\title{
An Anionic Nucleophilic Catalyst System for the Diastereoselective Synthesis of trans- $\beta$-Lactams
}

\author{
Anthony Weatherwax, Ciby J. Abraham, and Thomas Lectka* \\ Department of Chemistry, Johns Hopkins University, 3400 North Charles Street, \\ Baltimore, Maryland, 21218
}

General. Unless otherwise stated, all reactions were carried out under strictly anhydrous, air-free conditions. All reagents used were commercially available from Aldrich, Fluka and Acros Chemicals. All solvents and acid chlorides were dried and distilled by standard methods. ${ }^{1} \mathrm{H}$ and ${ }^{13} \mathrm{C}$ NMR spectra were acquired on a Bruker Avance $400 \mathrm{MHz}$ instrument in $\mathrm{CDCl}_{3}$. The ${ }^{1} \mathrm{H}(400 \mathrm{MHz})$ and ${ }^{13} \mathrm{C}(101 \mathrm{MHz})$ chemical shifts are given in parts per million $(\delta)$ with respect to internal TMS standard or residual solvent peaks. FTIR spectra were recorded on a Bruker Vector 22. The $\alpha$-imino ester $\mathbf{2}$ was synthesized from ethyl glyoxylate and $\mathrm{N}$ - $p$-toluenesulfonyl isocyanate by Weinreb's procedure. ${ }^{1}$ Catalyst $\mathbf{4 a}$ has been previously characterized. ${ }^{2}$

General Procedure for the Synthesis of $\boldsymbol{\beta}$-Lactams. To a solution of imidazoline derivative $4 \mathbf{b}(23 \mathrm{mg}, 0.031 \mathrm{mmol})$ and proton sponge 5 (76 $\mathrm{mg}, 0.312 \mathrm{mmol})$ in toluene $(5 \mathrm{~mL})$ at $0{ }^{\circ} \mathrm{C}$ was added phenylacetyl chloride $1 \mathbf{a}(48 \mathrm{mg}, 0.312 \mathrm{mmol})$ in toluene (1 $\mathrm{mL})$ followed by imino ester $2(240 \mathrm{mg}, 0.936 \mathrm{mmol})$ in toluene $(3 \mathrm{~mL})$. The reaction was maintained at $0{ }^{\circ} \mathrm{C}$ overnight before it was quenched with $1 \mathrm{M} \mathrm{HCl}(5 \mathrm{~mL})$ at $0{ }^{\circ} \mathrm{C}$. The aqueous layer was then extracted three times with $\mathrm{CH}_{2} \mathrm{Cl}_{2}$ and the combined organic layers were dried over $\mathrm{MgSO}_{4}$ and filtered through Celite. Absorption onto silica gel followed by column chromatography (10\% ethyl acetate/hexanes) afforded product 3a in $50 \%$ yield (58 $\mathrm{mg})$ and 37:1 dr [trans:cis].

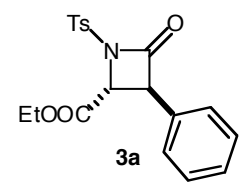

Trans-1-p-toluenesulfonyl-3-phenyl-4-ethoxycarbonylazetidinone (3a). White crystalline solid recrystallized from $\mathrm{Et}_{2} \mathrm{O} /$ hexanes: ${ }^{1} \mathrm{H} \mathrm{NMR}\left(\mathrm{CDCl}_{3}\right) ; \delta 7.98(\mathrm{~d}, 2 \mathrm{H}), 7.35$

\footnotetext{
${ }^{1}$ Tschaen, D. H.; Turos, E.; Weinreb, S. M. J. Org. Chem. 1984, 49, 5058.

${ }^{2}$ Matsura, T.; Ito, Y.; Saito, I. Bull. Chem. Soc. Jpn. 1973, 46, 3805-3809.
} 
$(\mathrm{d}, 4 \mathrm{H}), 7.22(\mathrm{~m}, 2 \mathrm{H}), 7.11(\mathrm{~m}, 1 \mathrm{H}), 4.62(\mathrm{~d}, 1 \mathrm{H}), 4.39(\mathrm{~d}, 1 \mathrm{H}), 4.30(\mathrm{~m}, 2 \mathrm{H}), 2.48(\mathrm{~s}$, $3 \mathrm{H}), 1.31(\mathrm{t}, 3 \mathrm{H}) \mathrm{ppm} ;{ }^{13} \mathrm{C} \mathrm{NMR}\left(\mathrm{CDCl}_{3}\right) \delta 168.2,163.0,145.9,135.5,131.9,130.0$, 129.5, 129.0, 128.2, 127.3, 62.5, 60.1, 59.1, 21.9, 14.4, ppm; IR $\left(\mathrm{CH}_{2} \mathrm{Cl}_{2}\right): 1803,1750$, 1516, 1370, 1254, 1172, 1089, $1032 \mathrm{~cm}^{-1}$. Anal. Calcd. for $\mathrm{C}_{19} \mathrm{H}_{19} \mathrm{NO}_{5} \mathrm{~S}: \mathrm{C}, 61.11 ; \mathrm{H}$, 5.13; N, 3.75. Found C, 61.23; H, 5.17; N, 3.76 .

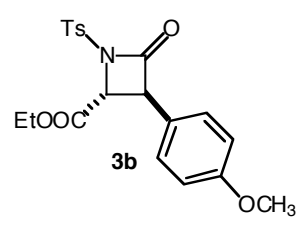

\section{Trans-1-p-toluenesulfonyl-3-(4-methoxyphenyl)-4-ethoxycarbonylazetidinone}

(3b). White crystalline solid recrystallized from $\mathrm{Et}_{2} \mathrm{O} /$ hexanes: ${ }^{1} \mathrm{H}$ NMR $\left(\mathrm{CDCl}_{3}\right) ; \delta 7.97$ $(\mathrm{d}, 2 \mathrm{H}), 7.39(\mathrm{~d}, 2 \mathrm{H}), 7.12(\mathrm{~d}, 2 \mathrm{H}), 6.87(\mathrm{~d}, 2 \mathrm{H}), 4.54(\mathrm{~d}, 1 \mathrm{H}), 4.32(\mathrm{~d}, 1 \mathrm{H}), 4.28(\mathrm{~m}, 2 \mathrm{H})$, 3.79 (s, 3H), 2.47 (s, 3H), 1.30 (t, 3H) ppm; ${ }^{13} \mathrm{C} \mathrm{NMR}\left(\mathrm{CDCl}_{3}\right) \delta 167.8,163.0,159.2$, 145.1, 134.8, 129.2, 128.0, 127.5, 123.3, 114.2, 61.7, 60.0, 58.3, 55.3, 21.1, 13.6 ppm; IR $\left(\mathrm{CH}_{2} \mathrm{Cl}_{2}\right): 1804,1751,1516,1370,1254,1172,1089,1033 \mathrm{~cm}^{-1}$. Anal. Calcd. for $\mathrm{C}_{20} \mathrm{H}_{21} \mathrm{NO}_{6} \mathrm{~S}: \mathrm{C}, 59.54 ; \mathrm{H}, 5.25 ; \mathrm{N}, 3.47$. Found C, 59.65; H, 5.28; N, 3.49 .



\section{Trans-1-p-toluenesulfonyl-3-(3-methoxyphenyl)-4-ethoxycarbonylazetidinone}

(3c). Yellowish oil, would not crystallize from $\mathrm{Et}_{2} \mathrm{O} /$ hexanes: ${ }^{1} \mathrm{H} \mathrm{NMR}\left(\mathrm{CDCl}_{3}\right) ; \delta 7.98$ $(\mathrm{d}, 2 \mathrm{H}), 7.39(\mathrm{~d}, 2 \mathrm{H}), 7.26(\mathrm{t}, 1 \mathrm{H}), 6.86(\mathrm{~d}, 1 \mathrm{H}), 6.79(\mathrm{~d}, 1 \mathrm{H}), 6.72(\mathrm{~s}, 1 \mathrm{H}), 4.60(\mathrm{~d}, 1 \mathrm{H})$, $4.36(\mathrm{~d}, 1 \mathrm{H}), 4.29(\mathrm{~m}, 2 \mathrm{H}), 3.78(\mathrm{~s}, 3 \mathrm{H}), 2.47(\mathrm{~s}, 3 \mathrm{H}), 1.31(\mathrm{t}, 3 \mathrm{H}) \mathrm{ppm} ;{ }^{13} \mathrm{C}$ NMR $\left(\mathrm{CDCl}_{3}\right) \delta 167.8,162.5,159.8,145.5,135.2,132.7,129.9,127.8,119.1,114.0,112.5$, 62.3, 59.7, 58.6, 55.1, 21.5, $13.8 \mathrm{ppm}$; IR $\left(\mathrm{CH}_{2} \mathrm{Cl}_{2}\right): 1802,1749,1373,1173,1088 \mathrm{~cm}^{-1}$. Anal. Calcd. for $\mathrm{C}_{20} \mathrm{H}_{21} \mathrm{NO}_{6} \mathrm{~S}: \mathrm{C}, 59.54 ; \mathrm{H}, 5.25 ; \mathrm{N}, 3.47$. Found C, 59.90; H, 5.59; N, 3.31 . 


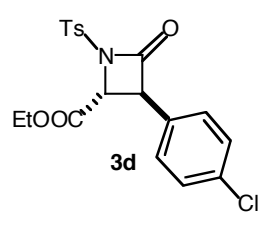

Trans-1-p-toluenesulfonyl-3-(4-chlorophenyl)-4-ethoxycarbonylazetidinone (3d). White crystalline solid recrystallized from $\mathrm{Et}_{2} \mathrm{O} /$ hexanes: ${ }^{1} \mathrm{H} \mathrm{NMR}\left(\mathrm{CDCl}_{3}\right) ; \delta 7.96(\mathrm{~d}$, 2H), $7.39(\mathrm{~d}, 2 \mathrm{H}), 7.33(\mathrm{~d}, 2 \mathrm{H}), 7.15(\mathrm{~d}, 2 \mathrm{H}), 4.57(\mathrm{~d}, 1 \mathrm{H}), 4.35(\mathrm{~d}, 1 \mathrm{H}), 4.29(\mathrm{~m}, 2 \mathrm{H})$, $2.47(\mathrm{~s}, 3 \mathrm{H}), 1.31(\mathrm{t}, 3 \mathrm{H}) \mathrm{ppm} ;{ }^{13} \mathrm{C} \mathrm{NMR}\left(\mathrm{CDCl}_{3}\right) \delta 168.3,162.7,146.1,135.6,130.4$, 129.9, 128.9, 127.4, 63.0, 60.3, 58.5, 22.1, 14.4 ppm; IR $\left(\mathrm{CH}_{2} \mathrm{Cl}_{2}\right): 1805,1751,1372$, 1189, 1091, $1016 \mathrm{~cm}^{-1}$. Anal. Calcd. for $\mathrm{C}_{19} \mathrm{H}_{19} \mathrm{ClNO}_{5} \mathrm{~S}: \mathrm{C}, 55.95 ; \mathrm{H}, 4.45 ; \mathrm{N}, 3.43$. Found C, 56.17; H, 4.48; N, 3.44.

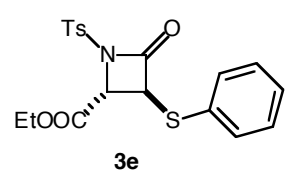

Trans-1-p-toluenesulfonyl-3-thiophenyl-4-ethoxycarbonylazetidinone (3e). White crystalline solid recrystallized from $\mathrm{Et}_{2} \mathrm{O} /$ hexanes: ${ }^{1} \mathrm{H} \mathrm{NMR}\left(\mathrm{CDCl}_{3}\right) ; \delta 7.76(\mathrm{~d}, 2 \mathrm{H}), 7.48$ $(\mathrm{d}, 2 \mathrm{H}), 7.30(\mathrm{~d}, 3 \mathrm{H}), 7.26(\mathrm{~d}, 2 \mathrm{H}), 4.32(\mathrm{~d}, 1 \mathrm{H}), 4.28(\mathrm{~d}, 1 \mathrm{H}), 4.25(\mathrm{~m}, 2 \mathrm{H}), 2.46(\mathrm{~s}, 3 \mathrm{H})$, $1.29(\mathrm{t}, 3 \mathrm{H}) \mathrm{ppm} ;{ }^{13} \mathrm{C} \mathrm{NMR}\left(\mathrm{CDCl}_{3}\right) \delta 169.8,167.2,161.0,146.0,145.3,134.4,129.6$, $129.4,129.3,127.5,62.3,58.8,57.1,21.5,14.7$ ppm; IR $\left(\mathrm{CH}_{2} \mathrm{Cl}_{2}\right): 1806,1748,1373$, $1174,1090,1026 \mathrm{~cm}^{-1}$. Anal. Calcd. for $\mathrm{C}_{19} \mathrm{H}_{19} \mathrm{NO}_{5} \mathrm{~S}_{2}$ : C, 56.28; H, 4.72; N, 3.45. Found C, $56.01 ; \mathrm{H}, 4.75 ; \mathrm{N}, 3.45$.

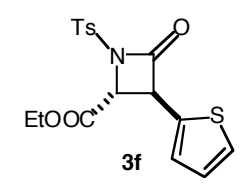

Trans-1-p-toluenesulfonyl-3-thiopheneyl-4-ethoxycarbonylazetidinone (3f). White crystalline solid recrystallized from $\mathrm{Et}_{2} \mathrm{O} /$ hexanes: ${ }^{1} \mathrm{H} \mathrm{NMR}\left(\mathrm{CDCl}_{3}\right) ; \delta 7.96(\mathrm{~d}, 2 \mathrm{H}), 7.39$ $(\mathrm{d}, 2 \mathrm{H}), 7.30(\mathrm{dd}, 1 \mathrm{H}), 6.98(\mathrm{~m}, 2 \mathrm{H}), 4.58(\mathrm{~d}, 1 \mathrm{H}), 4.56(\mathrm{~d}, 1 \mathrm{H}), 4.29(\mathrm{~m}, 2 \mathrm{H}), 2.48(\mathrm{~s}$, $3 \mathrm{H}), 1.32(\mathrm{t}, 3 \mathrm{H}) \mathrm{ppm} ;{ }^{13} \mathrm{C} \mathrm{NMR}\left(\mathrm{CDCl}_{3}\right) \delta 165.6,161.8,145.8,135.1,132.5,129.1$, 128.0, 127.4, 126.8, 126.4, 62.7, 61.9, 54.6, 21.7, 14.0 ppm; IR $\left(\mathrm{CH}_{2} \mathrm{Cl}_{2}\right): 1806,1752$, $1372,1172,1089,1024 \mathrm{~cm}^{-1}$. Anal. Calcd. for $\mathrm{C}_{17} \mathrm{H}_{17} \mathrm{NO}_{5} \mathrm{~S}_{2}: \mathrm{C}, 53.81 ; \mathrm{H}, 4.52 ; \mathrm{N}, 3.69$. Found C, 53.73; H, 4.44; N, 3.70. 


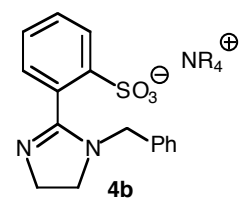

3-benzyl-2-(phenyl-2-sulfonate)-2-imidazoline tetraheptylammonium salt (4b). ${ }^{3}$ Sulfobenzoic anhydride $(1.000 \mathrm{~g}, 5.050 \mathrm{mmol})$, N-benzyl ethylenediamine $(0.782 \mathrm{~g}$, $5.050 \mathrm{mmol}$ ), and $20 \mathrm{~mL}$ of toluene were combined in a sealed tube and heated to $220{ }^{\circ} \mathrm{C}$ for $30 \mathrm{~h}$, then allowed to cool to RT. The reaction mixture was filtered and the solids washed thoroughly with toluene, and then recrystallized from $\mathrm{MeOH} / \mathrm{CH}_{2} \mathrm{Cl}_{2}$ to obtain 3benzyl-2-(phenyl-2-sulfonic acid)-2-imidazoline as a white powder $(1.170 \mathrm{~g}, 3.698$ mmol, 73\% yield). Tetraheptylammonium chloride (1.485 g, $3.161 \mathrm{mmol})$ was dissolved in $20 \mathrm{~mL} \mathrm{CH}_{2} \mathrm{Cl}_{2}$, to which was added $20 \mathrm{~mL}$ water. The previously prepared imidazoline derivative $(1.000 \mathrm{~g}, 3.161 \mathrm{mmol})$ was added to this solution as a solid and allowed to stir for $5 \mathrm{~min}$. With vigorous stirring, $6.3 \mathrm{~mL}$ of a $1 \mathrm{M} \mathrm{NaOH}$ solution was then added dropwise, and the reaction was stirred overnight. The solution is observed to go from cloudy to clear. Upon completion, the organics were drawn off and the aqueous extracted $5 \mathrm{X}$ with $10 \mathrm{~mL}$ portions of $\mathrm{CH}_{2} \mathrm{Cl}_{2}$. Combined all organics, dried briefly over $\mathrm{MgSO}_{4}$, filtered through Celite, then concentrated in vacuo to obtain the desired product $\left(1.9 \mathrm{~g}, 2.62 \mathrm{mmol}, 83 \%\right.$ yield) as a viscous yellow-orange oil: ${ }^{1} \mathrm{H}$ NMR (DMSO-d $\left.\mathrm{d}_{6}\right): \delta$ $7.83(\mathrm{dd}, 1 \mathrm{H}), 7.71(\mathrm{dd}, 1 \mathrm{H}), 7.51(\mathrm{~m}, 2 \mathrm{H}), 7.37(\mathrm{~d}, 2 \mathrm{H}), 7.32(\mathrm{t}, 2 \mathrm{H}), 7.24(\mathrm{t}, 1 \mathrm{H}), 3.73$ $(\mathrm{s}, 2 \mathrm{H}), 3.17(\mathrm{~m}, 12 \mathrm{H}), 2.88(\mathrm{t}, 2 \mathrm{H}), 2.73(\mathrm{t}, 2 \mathrm{H}), 1.57(\mathrm{~s}, 9), 1.29(\mathrm{~s}, 40), 0.88(\mathrm{t}, 15 \mathrm{H})$ ppm; ${ }^{13} \mathrm{C}$ NMR (DMSO-d ${ }_{6}$ ): $\delta$ 168.3, 144.2, 139.9, 131.5, 131.0, 130.7, 129.8, 128.4, 127.0, 126.8, 57.9, 52.3, 48.8, 45.6, 38.5, 31.1, 28.2, 25.9, 22.1, 21.1, $14.1 \mathrm{ppm}$; IR $\left(\mathrm{CH}_{2} \mathrm{Cl}_{2}\right): 2959,2930,2860,1457,1277,1256,1175 \mathrm{~cm}^{-1}$. (m/z): 410.7 (positive ion), 333.6 ( $\mathrm{n}+18$, negative ion). Anal. Calcd. for $\mathrm{C}_{16} \mathrm{H}_{19} \mathrm{~N}_{2} \mathrm{O}_{4} \mathrm{~S}:{ }^{4}$ C, 57.47; H, 5.43; N, 8.38. Found C, 57.16; H, 5.36; N, 8.34.

\section{Epimerization Studies}

General Procedure for Epimerization Studies. To cis-3a (20 mg, $0.054 \mathrm{mmol})$ in $4 \mathrm{~mL}$ toluene at $0{ }^{\circ} \mathrm{C}$ was added proton sponge $(11 \mathrm{mg}, 0.054 \mathrm{mmol})$ and catalyst $4 \mathbf{b}(4 \mathrm{mg}$, $0.005 \mathrm{mmol}$ ) together in $1 \mathrm{~mL}$ toluene. Let stir overnight, maintaining temperature at 0 ${ }^{\circ} \mathrm{C}$. Quenched with $1 \mathrm{M} \mathrm{HCl}(5 \mathrm{~mL})$ at $0{ }^{\circ} \mathrm{C}$. The aqueous layer was then extracted three times with $\mathrm{CH}_{2} \mathrm{Cl}_{2}$ and the combined organic layers were dried over $\mathrm{MgSO}_{4}$ and filtered

\footnotetext{
3 Synthesis adapted from Moehrke, H.; Koch, H.; von Freyberg, H. Verfahren zur Herstellung Heterocyclischer Verbindungen. Eur. Pat. Appl. DE865305, 1953.

${ }^{4}$ Microanalysis was conducted on the 3-benzyl-2-(phenyl-2-sulfonic acid)-2-imidazoline component of the catalytic system only and includes one mol equiv of water which was found to be irremovable even when drying under high vacuum over $\mathrm{P}_{2} \mathrm{O}_{5}$. The tetraheptylammonium portion was purchased from Acros and used as is. Due to a slight excess of the cation present, a ${ }^{1} \mathrm{H}$ NMR spectrum (see attached) is provided in lieu of microanalysis on the final, ion paired catalyst system.
} 
through Celite, then concentrated in vacuo. This crude reaction mixture was analyzed by NMR to determine the final $\mathrm{dr}$ of the $\beta$-lactam product.

The table below summarizes the results of these studies. Excluding trial 2, all tests were conducted at $0{ }^{\circ} \mathrm{C}$ and allowed to run overnight to mimic the conditions under which the lactams are formed, as discussed in our general $\beta$-lactam procedure above. Trial 2 was allowed to run at RT for 3 days to ascertain the equilibrium ratio of trans to cis diastereomers. Trial 4 suggests that PS by itself produces little epimerization, while catalyst $\mathbf{4 b}$ by itself (trial 3 ) causes the substrate to undergo only modest epimerization. When $\mathbf{4 b}$ and PS are combined (trials 1 and 2), a more significant shift in the dr is noted. However, the final observed dr, even at equilibrium, does not approach the level of selectivity observed in the products of our reactions (37:1 for lactam 3a), suggesting that the $\mathrm{dr}$ obtained in our experiments is not primarily due to epimerization of initially formed cis- $\beta$-lactam products. This evidence is further bolstered by trial 5 wherein a product mixture (3b) with an excess of the trans diastereomer was observed to lose selectivity upon further interaction with the catalyst and PS.

\begin{tabular}{|c|c|c|c|c|c|}
\hline Trial \# & Reagents & Rxn Time & Rxn Temp & Starting dr (t:c) & Final dr (t:c) \\
\hline 1 & $c i s-3 a, 4 b$, PS & overnight & $0^{\circ} \mathrm{C}$ & $1: 17$ & $1.4: 1$ \\
\hline 2 & $c i s-3 a, 4 b$, PS & 3 days & RT & $1: 17$ & $1.6: 1$ \\
\hline 3 & $c i s-3 a, 4 b$ & overnight & $0^{\circ} \mathrm{C}$ & $1: 17$ & $1: 1.7$ \\
\hline 4 & $c i s-3 a$, PS & overnight & $0^{\circ} \mathrm{C}$ & $1: 17$ & $1: 11$ \\
\hline 5 & $\mathbf{3 b}, \mathbf{4 b}$, PS & overnight & $0^{\circ} \mathrm{C}$ & $8.4: 1$ & $7.9: 1$ \\
\hline
\end{tabular}




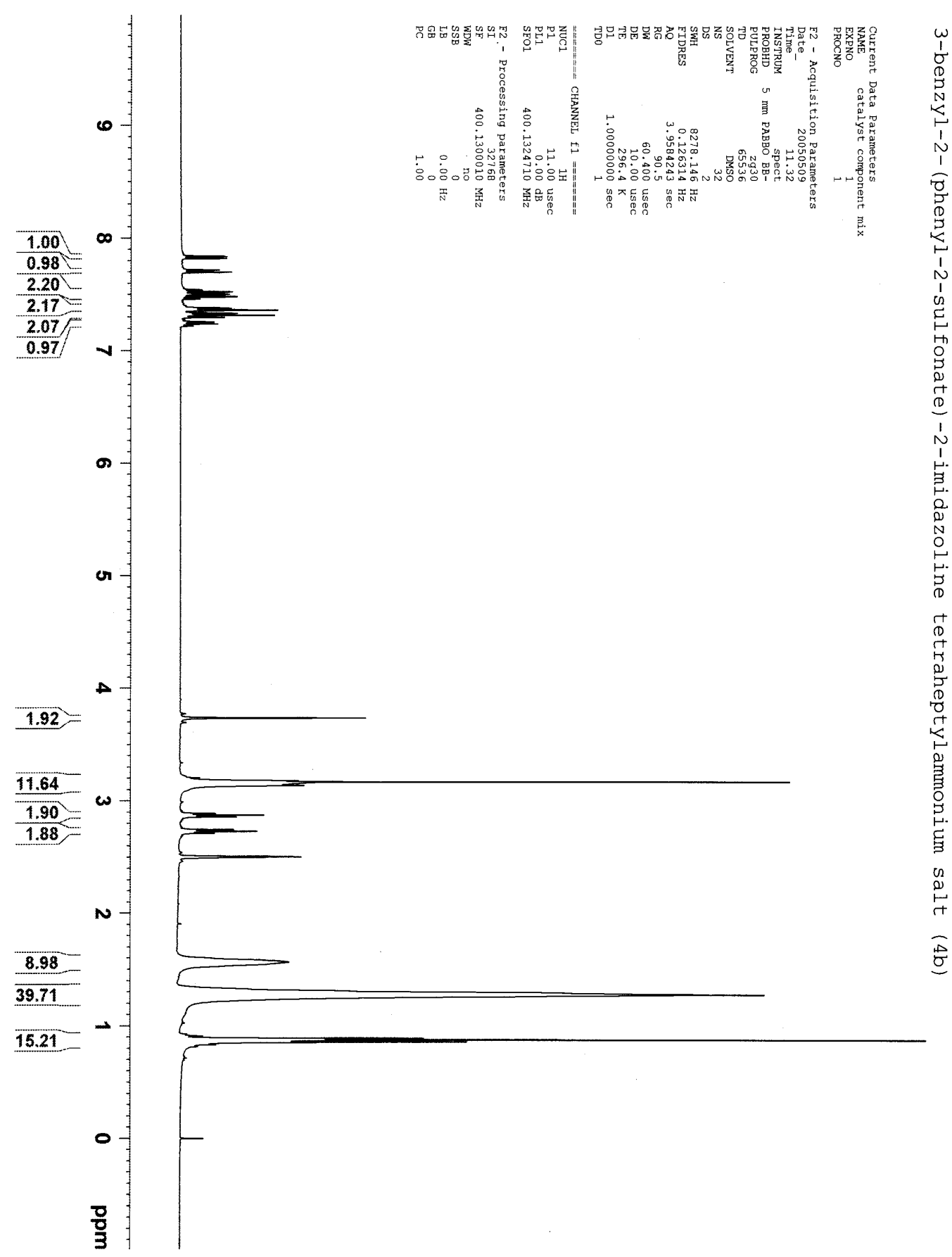

